\title{
Ergodic and Topological Properties of Coulombic Periodic Potentials
}

\author{
Andreas Knauf* \\ Institut für Theorie der Elementarteilchen, Arnimallee 14, D-1000 Berlin 33
}

\begin{abstract}
The motion of a classical pointlike particle in a two-dimensional periodic potential with negative coulombic singularities is examined. This motion is shown to be Bernoullian for many potentials and high enough energies. Then the motion on the plane is a diffusion process. All such motions are topologically conjugate and the periodic orbits can be analysed with the help of a group.
\end{abstract}

\section{Introduction}

As Markus and Meyer showed in [18], neither integrability nor ergodicity are generic properties of Hamiltonian systems with two degrees of freedom. Nevertheless, these extreme cases are much better understood than the complicated mixture inbetween. But, according to Berry "No smooth Hamiltonian of the type $H=$ kinetic + potential has been proven to be ergodic [7]." Though it is wellknown (see e.g. Anosov [2]) that the geodesic flow on a compact surface of strictly negative curvature is ergodic, this example is considered unphysical, since Efimov [11] showed that no such surface can be isometrically embedded in Euclidean 3-space.

One purpose of this paper is to show how such surfaces arise in the analysis of two-dimensional periodic potentials with negative coulombic singularities. This is the content of Sect. 2.

In Sect. 3 we give a new regularisation scheme for the collision orbits, which is well adapted to our purposes.

The measure-theoretical properties of the Hamiltonian motion are explored in Sect. 4. For a large class of Coulombic potentials the flow is shown to be Bernoullian. An account of the impacts of this notion is given by Ornstein [21]. We recall that another physical example of a Bernoulli system is given by the motion of a billard in a periodic array of convex obstacles, as shown by Gallavotti and Ornstein $[13,14]$.

^ Present address: Mathematisch instituut; Budapestlaan 6; NL-3508 TA Utrecht

This work is part of a thesis submitted to Freie Universität Berlin 
The diffusive behaviour of the particle moving in the plane is proven in Sect. 5 .

In Sect. 6 we deal with topological properties of the flow, complementing the measure-theoretical results. All systems with $n$ singularities in a unit cell are shown to be topologically conjugate. As a second result, a Morse-theoretic classification of all periodic orbits is given.

In the last section we speculate about related classical and quantum mechanical questions.

\section{Coulombic Potentials and Closed Riemannian Surfaces}

We start with the definition of the class of potentials we will consider. Let the lattice vectors $l_{1}, l_{2} \in \mathbb{C}$ be independent over $\mathbb{R}$ and define the lattice $\mathscr{C}$ as the set

$$
\mathscr{C}:=\left\{c \in \mathbb{C} \mid c=z_{1} l_{1}+z_{2} l_{2}, z_{1}, z_{2} \in \mathbb{Z}\right\} .
$$

Select as the fundamental domain $\mathscr{D}$ the set

$$
\mathscr{D}:=\left\{c \in \mathbb{C} \mid c=i_{1} l_{1}+i_{2} l_{2}, i_{1}, i_{2} \in[0,1[\} .\right.
$$

First fix the singularities by selecting $n$ different points $s_{i} \in \mathscr{D}, i \in\{1, \ldots, n\}$ and form the closed set

$$
\mathscr{S}:=\left\{c \in \mathbb{C} \mid c=s_{i}+l, l \in \mathscr{C}\right\} .
$$

We will consider the following class of periodic potentials on the punctured plane $M_{1}^{\prime}:=\mathbb{C} \backslash \mathscr{S}:$

Definition. Let $V: M_{1}^{\prime} \rightarrow \mathbb{R}$ be $C^{6}$ and periodic in $\mathscr{C}$, i.e. for $i=1,2$ and all $x \in M_{1}^{\prime} V\left(x+l_{i}\right)=V(x)$. If for all $i \in\{1, \ldots, n\}$ the limits

$$
c_{i}:=\lim _{x \rightarrow s_{i}} f_{i}(x)
$$

of the functions $f_{i}(x):=V(x)\left|x-s_{i}\right|$ exist, $c_{i}<0$, and all derivatives of $f_{i}(x)$ up to sixth order are bounded in some neighbourhood of $s_{i}$, then $V$ is called (negative) coulombic.

As a first step we observe that by the very definition of a coulombic potential $V$ the maximal potential energy

$$
h_{0}:=\sup _{x \in M_{1}^{\prime}} V(x)
$$

is finite.

So instead of considering the dynamical system given by the Hamiltonian $H=\frac{1}{2}\left(p_{1}^{2}+p_{2}^{2}\right)+V\left(q_{1}+i q_{2}\right), p_{1}, p_{2}, q_{1}, q_{2} \in \mathbb{R}$, with energies $H=h>h_{0}$, we may equivalently discuss the geodesic motion on $\left(M_{1}^{\prime}, g_{1}^{\prime}\right)$ with Jacobi metric

$$
g_{1}^{\prime}(x):=(h-V(x)) \cdot g(x)
$$

which is conformally equivalent to the Euclidean metric $g(x)$ on $M_{1}^{\prime}$ (see, e.g. Abraham and Marsden [1, Chap. 3.7]).

Now the length $L(\sigma)$ of a curve $\sigma:] a, b\left[\rightarrow M_{1}^{\prime}\right.$ is given by

$$
L(\sigma):=\int_{a}^{b}\|\dot{\sigma}(t)\| d t
$$


with the $g_{1}^{\prime}$-norm $\|\cdot\|$. For a curve

$$
\sigma:] 0,1\left[\rightarrow M_{1}^{\prime}, \quad \sigma(t):=s_{i}+c \cdot t,\right.
$$

approaching one singularity with $c$ small, one sees that $L(\sigma)<\infty$ using $L(\sigma)$ $<k \int_{0}^{1} t^{-1 / 2} d t$ for some $k>0$. So $\left(M_{1}^{\prime}, g_{1}^{\prime}\right)$ is incomplete. Nevertheless, a direct consequence of Theorem 1 will be some sort of a Hopf-Rinow theorem, i.e. any two points $p, q \in M_{1}^{\prime}$ will be connected by a minimal geodesic in $M_{1}^{\prime}$. However, $M_{1}^{\prime}$ cannot be completed in a direct way, by adding some limit points.

To obtain a first insight into the singularity structure, we consider a family $\gamma_{r}: S^{1} \rightarrow M_{1}^{\prime}$ of circular loops with winding number 1 around $s_{i}$, parametrized by their Euclidean radius $r>0$. Integrating the geodesic curvature $k_{g}$ of the loops, we find that

$$
\lim _{r \rightarrow 0} \oint_{S^{1}} k_{g}(t) d t=\pi \text {. }
$$

In absence of a singularity this limit would take the value $2 \pi$. So an isometric embedding of some circular neighbourhood $U$ of a singularity $s_{i}$ in $\mathbb{R}^{3}$ roughly looks like a cone with an aperture of $\pi / 6$.

By the following construction we can regularize the singularity. We cut $U$ radially to obtain one half of a disc. Pasting this surface together along the banks of the cut with a second copy of itself, we get a circular disk $U^{\prime}$. Now a loop in $U^{\prime}$ surrounding the former singularity once has the (limiting) geodesic curvature of $2 \cdot \pi$ which means that the covering surface $U^{\prime}$ has no singular point.

Now one can find complex coordinates $z$ for $U^{\prime}$ and $w$ for $U$ such that the above branched covering construction has the analytical form $w=z^{2}$. This construction is a geometrical reinterpretation of the so-called Levi-Civita Transformation (see e.g. Stiefel and Scheifele [30]).

Consider next the torus $M_{2}:=\mathbb{C} / \mathscr{C}$ and its subset $M_{2}^{\prime}:=M_{1}^{\prime} / \mathscr{C}$ with the induced covering $\pi_{12}: M_{1} \rightarrow M_{2}$. Since there is no danger of confusion, we will denote the projected singularities $\pi_{12}\left(s_{i}\right)$ by $s_{i}$, too. The metric $g_{1}^{\prime}$ on $M_{1}^{\prime}$ is projectable and thus $g_{2}^{\prime}:=\pi_{12 *} g_{1}^{\prime}$ is unambiguously defined on $M_{2}^{\prime}$.

$M_{2} \cong \mathbb{T}^{2}$ has Euler characteristic 0 . Using the expression above for the limit of the geodesic curvature of a loop around a singularity, which clearly is valid for $\left(M_{2}^{\prime}, g_{2}^{\prime}\right)$, too, and the Gauss-Bonnet theorem, the integral of the Gaussian curvature $K(x)$ is seen to equal

$$
\int_{M_{2}^{\prime}} K(x) d M_{2}=-n \cdot \pi
$$

So we have the chance to find coulombic potentials leading to Riemannian manifolds whose Gaussian curvature is negative at all points. The geodesic motion on such manifolds is known to be ergodic.

Now we shall transform the dynamical problem on $M_{2}^{\prime}$ into one on a complete, compact Riemannian surface $M_{4}$ which will be a branched covering of $M_{2}$.

Theorem 1. There exists a compact Riemannian surface $M_{4}$ with a Riemannian metric $\mathrm{g}_{4}$ ( six times continuously differentiable) having genus $\mathscr{G}\left(M_{4}\right)=2 n+1$, and a 8-sheeted branched covering $\pi_{42}: M_{4} \rightarrow M_{2}$ with branch points $\left\{\pi_{42}^{-1}\left(s_{i}\right)\right\} \subset M_{4}$ and 
branch numbers $b_{\pi_{42}}(x)=1$ for all branch points $x$, such that $\pi_{42}$ is a local isometry on $M_{4}^{\prime}:=M_{4} \backslash\{$ branch points $\}$.

Proof. Clearly one has to perform the Levi-Civita transformation near every point $s_{i} \in M_{2}$. But to globalize this transformation and to find $M_{4}$ we first define an auxiliary torus $M_{3}$ covering the torus $M_{2}$ four times.

So consider the lattice

$$
\mathscr{C}_{2}:=\left\{c \in \mathbb{C} \mid c=2 \cdot\left(z_{1} l_{1}+z_{2} l_{2}\right), z_{1}, z_{2} \in \mathbb{Z}\right\} .
$$

Set $M_{3}:=\mathbb{C} / \mathscr{C}_{2}$ and let $\pi_{13}: M_{1} \rightarrow M_{3}$ be the induced covering. Define

$$
\begin{gathered}
s_{i}^{\mathrm{I}}:=\pi_{13}\left(s_{i}\right), \quad s_{i}^{\mathrm{II}}:=\pi_{13}\left(s_{i}+l_{1}\right), \\
s_{i}^{\mathrm{III}}:=\pi_{13}\left(s_{i}+l_{2}\right) \quad \text { and } \quad s_{i}^{\mathrm{IV}}:=\pi_{13}\left(s_{i}+l_{1}+l_{2}\right)
\end{gathered}
$$

for $i \in\{1, \ldots, n\}$. These points are pairwise different (see Fig. 1).

Recall that we visualized the Levi-Civita transformation by radially cutting a circular neighbourhood of a singularity. To globalize the transformation we now cut $M_{3}$ along the curves $c_{i}^{A}$ connecting $s_{i}^{\mathrm{I}}$ with $s_{i}^{\mathrm{III}}$ respectively $c_{i}^{B}$ connecting $s_{i}^{\mathrm{II}}$ with $s_{i}^{\mathrm{IV}}$.

As in the local case we then paste two copies of the cut tori together along banks of corresponding cuts yielding the manifold $M_{4}$. (Observe, however, that the left banks on one torus are connected to the right banks on the other one. So there are selfintersections in this 3-dimensional immersion.)

To define $M_{4}$ more formally, we observe that there exists a doubly periodic meromorphic (=elliptic) function $f$ with order one zeroes at $s_{i}^{\mathrm{III}}$ and $s_{i}^{\mathrm{IV}}$ and order one poles at $s_{i}^{\mathrm{I}}$ and $s_{i}^{\mathrm{II}}$. Although we shall not need its explicit form (its existence is granted by Abels theorem using the 1-chain $\left.c:=\sum_{i=1}^{n}\left(c_{i}^{A}+c_{i}^{B}\right)\right)$, it is given uniquely (up to a constant factor) in terms of the Weierstrass $\sigma$-function (see, e.g. Behnke and Sommer [6]),

$$
\begin{gathered}
\sigma(z):=z \prod_{w \in \mathscr{C}_{2} \backslash\{0\}}\left(1-\frac{z}{w}\right) \exp \left(\frac{z}{w}+\frac{1}{2}\left(\frac{z}{w}\right)^{2}\right), \\
f(z):=\frac{\sigma\left(z-s_{1}^{\mathrm{III}}\right) \sigma\left(z-s_{1}^{\mathrm{IV}}\right) \ldots \sigma\left(z-s_{n}^{\mathrm{III}}\right) \sigma\left(z-s_{n}^{\mathrm{IV}}\right)}{\sigma\left(z-s_{1}^{\mathrm{I}}\right) \sigma\left(z-s_{1}^{\mathrm{II}}\right) \ldots \sigma\left(z-s_{n}^{\mathrm{I}}\right) \sigma\left(z-s_{n}^{\mathrm{II}}\right)} .
\end{gathered}
$$

Fig. 1. The 1-chain $c$

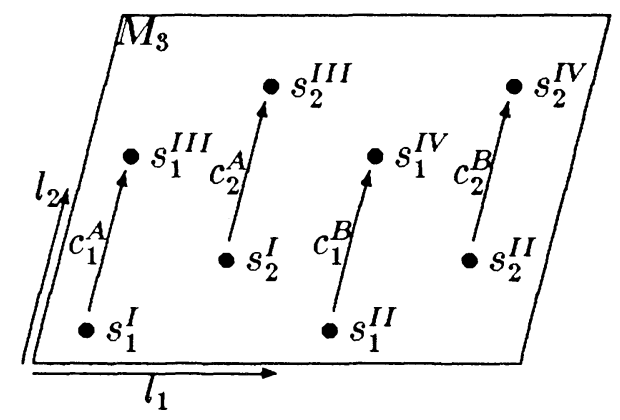


Since $f(x+w)=f(x)$ for $w \in \mathscr{C}_{2}, f$ projects to a meromorphic function on the torus $M_{3}$, which we denote by $f$, too.

Now consider the concrete Riemann surface $M_{4}$ given by the equation

$$
u^{2}=f(v), \quad u \in \mathbb{C} \cup \infty, \quad v \in M_{3} .
$$

The branched covering $\pi_{43}: M_{4} \rightarrow M_{3}$ is given by projection on the $v$ variable of the defining relation. Clearly $\pi_{43}$ is $k=2$-sheeted with branch points $\hat{s}_{i}^{r}:=\pi_{43}^{-1}\left(s_{i}^{r}\right)$, $r \in\{I, I I, I I I, I V\}$ and branch numbers $b_{\pi_{43}}\left(\hat{s}_{i}^{r}\right)=1$ for all branch points. So the total branching number $B$ equals $4 n$.

From the Riemann-Hurwitz relation for the genus one obtains

$$
\mathscr{G}\left(M_{4}\right)=\frac{B}{2}+k\left(\mathscr{G}\left(M_{3}\right)-1\right)+1=2 n+1 .
$$

Since $\pi_{32}: M_{3} \rightarrow M_{2}$ defined by $\pi_{32}:=\pi_{12} \circ \pi_{13}^{-1}$ is a four sheeted (unbranched) covering, $\pi_{42}: M_{4} \rightarrow M_{2}$ given by $\pi_{42}:=\pi_{32} \circ \pi_{43}$ is an eight sheeted branched covering of $M_{2}$ with branch points $\hat{S}_{i}^{r}$.

Now we define a metric $g_{4}$ on $M_{4}$ in two steps. First we lift the metric $g_{2}^{\prime}$ defined on $M_{2}^{\prime}$ to $M_{4}^{\prime}:=M_{4} \backslash\left\{\hat{s}_{i}^{\prime}\right\}$, i.e. everywhere except at the branch points, by setting $g_{4}^{\prime}(x):=g_{2}^{\prime}\left(\pi_{42}(x)\right)$ for $x \in M_{4}^{\prime}$. Thus the projection $\pi_{42}$ becomes a local isometry from $M_{4}^{\prime}$ to $M_{2}^{\prime}$.

Secondly we define the metric $g_{4}$ at the branch points $\hat{S}_{i}^{r}$. We only have to repeat the Levi-Civita construction outlined above in a more formal manner.

For $\hat{s}_{i}^{r} \in M_{4}$ projecting to $s_{i} \in M_{2}$ we use a chart $\left(U_{2}, \phi_{2}\right), s_{i} \subset U_{2} \in M_{2}$ which is an affine translation of the complex coordinates in the plane with origin at $s_{i}[i . e$. $\phi_{2}\left(s_{i}\right)=0$ and $\left.V\left(\pi_{12}^{-1}\left(\phi_{2}^{-1}(w)\right)\right)=V\left(s_{i}+w\right)\right]$. Since the elliptic function $f$ has only first order zeroes and poles, we can find a local chart $\left(U_{4}, \phi_{4}\right), \hat{s}_{i}^{r} \subset U_{4} \in M_{4}$ with $\phi_{4}\left(\hat{s}_{i}^{r}\right)=0$ which has the property that the projection $\pi_{42}$ attains exactly the simple form of the Levi-Civita transformation:

$$
\phi_{2} \circ \pi_{42} \circ \phi_{4}^{-1}(z)=z^{2}
$$

(see Farkas and $\mathrm{Kra}[12$, I.1.6] for the simple construction of such a chart). Clearly this special choice of charts has been made just to simplify the calculations.

Now we extend the metric $g_{4}^{\prime}$ on $M_{4}^{\prime}$ to a metric $g_{4}$ on $M_{4}$ in the following way. To define $g_{4}$ at $\hat{s}_{i}^{r}$, we perform the limit $z \rightarrow 0$ for its analytic expression $g_{4}^{\text {Ch. }}(z)$ in the local chart $\left(U_{4}, \phi_{4}\right)$.

The line elements in the two charts are

$$
d s^{2}=g_{2}^{\text {Ch. }}(w) d w d \bar{w}=g_{4}^{\text {Ch. }}(z) d z d \bar{z},
$$

and we can substitute $z^{2}=w \neq 0$.

Thus we find

$$
g_{4}^{\text {Ch. }}(z)=4 z \bar{z} g_{2}^{\text {Ch. }}\left(z^{2}\right)=4 z \bar{z}\left(h-V\left(s_{i}+z^{2}\right)\right)=4\left(h z \bar{z}-f_{i}\left(s_{i}+z^{2}\right)\right),
$$

with $f_{i}(x)$ defined in the above definition of coulombic potentials. Now

$$
g_{4}^{\mathrm{Ch}}(0)=\lim _{z \rightarrow 0} g_{4}^{\mathrm{Ch}}(z)=-4 \cdot f_{i}\left(s_{i}\right)=-4 \cdot c_{i}>0
$$

and by the assumptions on the coulombic potential $V$ it is $C^{6}$.

So the metric $g_{4}$ is $C^{6}$ on the whole of $M_{4}$. 
Remarks. 1. $M_{2}$ is a so-called $V$-manifold (orbifold) in the sense of Satake $[25,26]$. This can be seen using the above definition of $M_{4}$ : a group $G=\mathbb{Z}_{2} \times \mathbb{Z}_{2} \times \mathbb{Z}_{2}$ acts on $M_{4}$ by isometric covering transformations $\Psi_{g}: M_{4} \rightarrow M_{4}, g \in G$ which leave the projection invariant: $\pi_{42}\left(\Psi_{g}(x)\right)=\pi_{42}(x) . M_{2}$ equals $M_{4} / G$. The singular points $s_{i}$ arise as isolated fixed points of isometries $\Psi_{g}$. Therefore $M_{4} / G$ has a canonical $V$-manifold structure [26].

2. In the context of complex function theory the use of the auxiliary fourfold covering $\pi_{32}: M_{3} \rightarrow M_{2}$ cannot be avoided since there does not exist an elliptic function with just one first order pole in its fundamental parallelogram (see e.g. Behnke and Sommer [6, p. 137]).

\section{Regularization}

Owing to the possible collisions with the singularities $s_{i}$ the geodesic flow in the unit tangent bundle $T_{1} M_{2}^{\prime}$ of the surface $\left(M_{2}^{\prime}, g_{2}^{\prime}\right)$ is incomplete.

Our strategy will be to regularize the flow on the fundamental domain (and on the whole plane) using the completeness of the geodesic flow in the unit tangent bundle $T_{1} M_{4}$ of $\left(M_{4}, g_{4}\right)$. One necessary step is to compactify $T_{1} M_{2}^{\prime}$ by adding $n$ circles, one for each singularity $s_{i}$ in $M_{2}$. As it will turn out, the compactified space $X$ will be different from $T_{1} M_{2}$, i.e. the circles will be added in a nontrivial way.

In celestial mechanics, various regularisation schemes have been invented to complete the so-called collision orbits $[10,28,30]$. The reason why we give another equivalent regularisation is simply that it seems to fit more directly in our context. To be specific, we will see that $T_{1} M_{4}$ is an unbranched covering of $X$ and the flow on $X$ is just the projection of the flow on $T_{1} M_{4}$. This will describe the physical situation by which the mass point is reflected backwards at the singularities.

Since $\pi_{42}: M_{4} \rightarrow M_{2}$ is a branched covering, the projection of the geodesic lines is singular at each point $s_{i} \in M_{2}$. This is depicted in Fig. 2. By definition of $g_{4}$ the (incomplete) geodesic flow on $\left(M_{2}^{\prime}, g_{2}^{\prime}\right)$ equals the projected flow in the unit tangent bundle $T_{1} M_{4}^{\prime}$ using the push-forward $\pi_{42 *}$ from $T_{1} M_{4}^{\prime}$ to $T_{1} M_{2}^{\prime}$.

In the last section we defined the $V$-manifold structure of $M_{2}$ using the action $\Psi_{g}$ of a group $G$ of isometries on $\left(M_{4}, g_{4}\right): M_{2}=M_{4} / G$. Now the push-forward $\Psi_{g *}$ acts on the unit tangent bundle: $\Psi_{g *}: T_{1} M_{4} \rightarrow T_{1} M_{4}$. So it is natural to define $X$ by

$$
X:=T_{1} M_{4} / G
$$

using this action, and we denote by $\tilde{\pi}_{42 *}: T_{1} M_{4} \rightarrow X$ the induced projection.

Clearly $T_{1} M_{4}^{\prime} / G$ equals $T_{1} M_{2}^{\prime}$, so that we have an inclusion of the noncompact space $T_{1} M_{2}^{\prime}$ in $X$.

Fig. 2. Geodesic lines in $M_{4}$ (left) project to hyperbolae in $M_{2}$ (right)

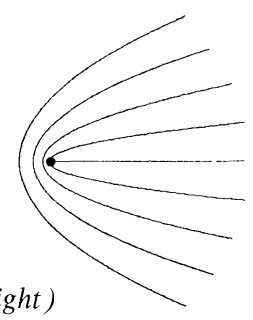


One should expect that $X$, due to the singularities shown in Fig. 2, is a $V$-manifold without a differentiable structure.

Surprisingly, however, $X$ is a manifold in the ordinary sense:

Theorem 2. $X=T_{1} M_{4} / G$ is a compact three dimensional differentiable manifold. $\tilde{\pi}_{42 *}: T_{1} M_{4} \rightarrow X$ is an eightfold (unbranched) covering. The regularized geodesic flow $\Phi_{X}: \mathbb{R} \times X \rightarrow X$ in $X$ is uniquely defined by

for $x \in T_{1} M_{4}$.

$$
\Phi_{X}\left(t, \tilde{\pi}_{42 *}(x)\right):=\tilde{\pi}_{42 *}\left(\Phi_{4}(t, x)\right)
$$

Proof. $\Psi_{g *}$ acts freely on $T_{1} M_{4}$, i.e. for each $x \in T_{1} M_{4}$ the map $g \mapsto \Psi_{g *}(x)$ is oneto-one. This is clear unless $x$ is a unit tangent vector at a singular point $\hat{S}_{i}^{r} \in M_{4}$. But then for any group element $g \neq e$ with $\Psi_{g}\left(\hat{S}_{i}^{r}\right)=\hat{s}_{i}^{r}$ still $x$ is not a fixed point of $\Psi_{g *}$, since its direction is inverted.

Since $G$ is a finite group (of order 8 ) and thus its action $\Psi_{g *}$ on $T_{1} M_{4}$ is proper, by Proposition 4.1.23 of [1] $X$ is a smooth manifold, and $\tilde{\pi}_{42 *}$ is a submersion. By construction $\Phi_{X}$ is a differentiable flow in $X$ which coincides on $T_{1} M_{2}^{\prime} \subset X$ with the incomplete geodesic flow of the metric $g_{2}^{\prime}$.

Remark. Since $\left(M_{1}^{\prime}, g_{1}^{\prime}\right)$ is locally isometric to $\left(M_{2}^{\prime}, g_{2}^{\prime}\right)$ via $\pi_{12}$, one can define a complete flow $\Phi_{Y}: \mathbb{R} \times Y \rightarrow Y$ on the completion $Y$ of $T_{1} M_{1}^{\prime}$, which describes the motion of the particle on the whole plane, including collision orbits. Then by extending the push forward projection

$$
\pi_{12 *}: T_{1} M_{1}^{\prime} \rightarrow T_{1} M_{2}^{\prime}
$$

one obtains an unbranched covering $\pi_{Y X}: Y \rightarrow X$ such that the flow $\Phi_{Y}$ projects to $\Phi_{X}$.

\section{Ergodic Properties}

It is well known that the properties of the geodesic flow on a compact manifold depend to a large degree on the sign of its sectional curvature. So our first step for proving ergodic properties is to express the curvature of $\left(M_{4}, g_{4}\right)$ in terms of the potential $V$, the Gaussian curvature being equal to the sectional curvature in the two dimensional case. Gauss' theorema egregium [16] says:

$$
K(x)=\frac{R_{1212}(x)}{\operatorname{det}\left(g_{i k}(x)\right)} .
$$

For conformal metrics $g_{i k}(x)=F^{-2}(x) \delta_{i k}$, one has

$$
\begin{gathered}
R_{j i j}^{i}=\frac{\partial^{2} f}{\partial x^{j} \partial x^{j}}+\frac{\partial^{2} f}{\partial x^{i} \partial x^{i}}-\sum_{r \neq i, j}\left(\frac{\partial f}{\partial x^{r}}\right)^{2}, \\
i \neq j \text { with } f(x):=\log F(x),
\end{gathered}
$$

see e.g. Spivak [29]. In our case $f(x)=-\frac{1}{2} \log (h-V(x))$, so

$$
K(x)=\frac{(h-V(x)) \Delta V(x)+(V V(x))^{2}}{(h-V(x))^{3}}
$$

with the gradient $\nabla$ and the two-dimensional Euclidean Laplacian $\Delta$. 
Definition. Let $V: M_{1}^{\prime} \rightarrow \mathbb{R}$ be a coulombic potential and $g_{1}^{\prime}(x)=(h-V(x)) g(x)$ the metric on $M_{1}^{\prime}$ defined by $V$. If the Gaussian curvature $K(x)$ of $\left(M_{1}^{\prime}, g_{1}^{\prime}\right)$ is $\leqq 0$ and on every maximally extended geodesic on $M_{1}^{\prime}$ there exist points $x$ with $K(x)<0$, then $V$ is said to be of negative curvature at the energy $h$.

The condition on the curvature along geodesics resembles what was called a "finite horizon" in the context of ergodic billard motion [9]. This is a slight extension compared to the standard notion of strictly negative curvature. So we have to prove the existence of some exponential bound for the separation of trajectories in the (un)stable submanifolds of the tangent space $T T_{1} M_{4}$ of the unit tangent bundle $T_{1} M_{4}$ to obtain the Anosov property.

The following lemma is analogous to Lemma A 21.17 in Arnold and Avez [5].

Lemma 1. Let $s$ be an even natural number, $t_{0} \in \mathbb{R}^{+}$fixed and $r: \mathbb{R}^{+} \rightarrow \mathbb{R}$ be a $C^{2}$-differentiable function. Let $r(0)>0, r\left(s t_{0}\right)=0$, and for $0 \leqq t \leqq s t_{0}$,

$$
\ddot{r}(t) \cdot r(t) \geqq k^{2}(t) r^{2}(t)
$$

for a function $k(t)$ with

$$
\int_{u}^{u+t_{0}} k^{2}(t) d t \geqq C^{2}>0 \quad \text { for all } u .
$$

Then for $t \in\left[0, s t_{0}\right]$ and $k_{0}=\frac{1}{2} \log \left(1+C^{2} t_{0}\right)$,

$$
\begin{gathered}
0 \leqq r(t) \leqq r(0) \exp \left(2 k_{0}\right) \exp \left(-\frac{k_{0} t}{t_{0}}\right), \\
|\dot{r}(t)| \leqq|\dot{r}(0)| \exp \left(2 k_{0}\right) \exp \left(-\frac{k_{0} t}{t_{0}}\right) .
\end{gathered}
$$

Proof. First, observe that $r(t) \geqq 0$ for $t \in\left[0, s t_{0}\right]$ since otherwise there would exist a negative value $r\left(t^{\prime}\right)<0$ with $\ddot{r}\left(t^{\prime}\right)<0$. So $\ddot{r}(t) \geqq 0$ for $t \in\left[0, s t_{0}\right]$ such that $\dot{r}(t)$ increases. Since $\dot{r}\left(s t_{0}\right) \leqq 0$, the slope $\dot{r}(t) \leqq 0$ in the whole interval.

So the inequality $r(t-u) \geqq r(t)-u \dot{r}(t)$ is valid for $0 \leqq u \leqq t \leqq s t_{0}$. For $\dot{r}(t)$ we get a second inequality $(n \in\{1,2, \ldots, s\})$ as follows:

$$
\begin{aligned}
\dot{r}\left((s-n) t_{0}\right)-\dot{r}\left((s-n+1) t_{0}\right) & =-\int_{(s-n) t_{0}}^{(s-n+1) t_{0}} \ddot{r}(t) d t \\
& \leqq-\int_{(s-n) t_{0}}^{(s-n+1) t_{0}} k^{2}(t) r(t) d t \\
& \leqq-\int_{(s-n) t_{0}}^{(s-n+1) t_{0}} k^{2}(t) r\left((s-n+1) t_{0}\right) d t \\
& \leqq-C^{2} r\left((s-n+1) t_{0}\right) .
\end{aligned}
$$

Iterating these inequalities, one obtains:

$$
\begin{aligned}
& r\left((s-n) t_{0}\right) \geqq r\left((s-n+2) t_{0}\right)\left(1+C^{2} t_{0}\right)-2 t_{0} \dot{r}\left((s-n+2) t_{0}\right), \\
& \dot{r}\left((s-n) t_{0}\right) \leqq \dot{r}\left((s-n+2) t_{0}\right)\left(1+C^{2} t_{0}\right)-2 C^{2} r\left((s-n+2) t_{0}\right) .
\end{aligned}
$$


So we get

$$
\begin{gathered}
r\left(2 m t_{0}\right) \leqq r(0)\left(1+C^{2} t_{0}\right)^{-m}, \quad\left|\dot{r}\left(2 m t_{0}\right)\right| \leqq|\dot{r}(0)|\left(1+C^{2} t_{0}\right)^{-m}, \\
(m \in\{0,1, \ldots, s / 2\}) .
\end{gathered}
$$

Now $\left(1+C^{2} t_{0}\right)^{-m}=\exp \left(-m \log \left(1+C^{2} t_{0}\right)\right)$, and using the monotonicity, we get the stated inequalities.

Let $\mu_{X}$ denote the invariant natural volume on $X$.

Theorem 3. If the coulombic potential $V$ is of negative curvature at energy $h$, then the flow $\Phi_{X}$ on $\left(X, \mu_{X}\right)$ is an Anosov and Bernoulli flow.

Proof. We must invoke Lemma 2 with the negative Gaussian curvature $-K\left(\gamma_{4}(t)\right)$ along a geodesic $\gamma_{4}$ in $M_{4}$ playing the rôle of $k^{2}(t)$. As in [5] we can use Lemma 2 to show the existence of geodesics whose distance to any given geodesic contracts exponentially in positive time.

We first show that the conditions of Lemma 2 are fulfilled, i.e. that there exists a $t_{0}>0$ and a $C>0$ such that we have the estimate

$$
-\int_{0}^{t_{0}} K\left(\gamma_{4}(t)\right) d t \geqq C^{2}
$$

for every geodesic $\gamma_{4}(t)$ in $M_{4}$. Assume the contrary. Then by compactness of $T_{1} M_{4}$ there must exist a geodesic $\gamma_{4}(t)$ in $M_{4}$ such that

$$
\int_{0}^{\infty} K\left(\gamma_{4}(t)\right) d t=0
$$

Hence $\gamma_{4}(t)$ must be a projection of a geodesic in $\mathbb{C}$ under $\pi_{43}^{-1} \circ \pi_{13}: \mathbb{C} \rightarrow M_{4}$. But we required that on every such geodesic there exist points $x$ with $K(x)<0$.

So the conditions of Lemma 2 are fulfilled.

Lemma 2 certainly does not give the best possible exponential bounds, but it suffices to extend the Lobatchewski-Hadamard theorem (see, e.g. [5]) to our case. As a consequence $\Phi_{4}$ on $T_{1} M_{4}$ and $\Phi_{X}$ on $X$ are Anosov flows.

Not every Anosov flow is Bernoulli. But Theorem 3 of Ornstein and Weiss [22] states that the geodesic flow on a compact $C^{2}$-manifold of strictly negative curvature with natural volume measure is isomorphic to a Bernoulli flow. Ornstein (see Brown [8]) proved that every factor of a Bernoulli system is a Bernoulli system. But $\tilde{\pi}_{42 *}: T_{1} M_{4} \rightarrow X$ is a measure preserving map and the diagram

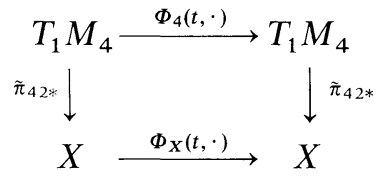

commutes for all $t$ by Theorem 2. So $X$ is a factor of $T_{1} M_{4}$ for all $t$ and so $\Phi_{X}$ is isomorphic to a Bernoulli flow, too.

Remarks. 1. The main idea put forward in this section is quite old. See the footnote on p. 743 of the article of A. N. Kolmogorov reprinted in [1].

2. $\Phi_{X}$ is also a $\mathbb{K}$-system, mixing, and ergodic, as follows from the ergodic hierarchy. 
3. Since the geodesic flow $\Phi_{4}$ on $T_{1} M_{4}$ is of Anosov type, standard results as for example Theorem 5.4.3 of Klingenberg [17] are valid. See Remark 2 following Theorem 6 below.

Now we will show that the conditions of Theorem 3 are met in the cases of Yukawa potentials and certain coulombic potentials of finite range.

\subsection{Example 1: Yukawa Potentials}

Let the multiindex $I:=\left(i_{1}, i_{2}, i_{3}\right)$ vary in $\{1, \ldots, n\} \times \mathbb{Z} \times \mathbb{Z}$. We consider $n$ singularities of strengths $c_{i_{1}}$ at different points $s_{i_{1}}$ in the fundamental domain $\mathscr{D}\left(c_{i_{1}}<0\right)$. The points $x_{I} \in \mathscr{S}$ in the complex plane are defined by $x_{I}=s_{i_{1}}$ $+i_{2} l_{1}+i_{3} l_{2}$. The periodic Yukawa potential on $M_{1}^{\prime}$ is given by

$$
V(x):=\sum_{I} c_{i_{1}} \frac{\exp \left(-\mu_{i_{1}} r_{I}\right)}{r_{I}},
$$

where $r_{I}$ is the abbreviation for $\left|x-x_{I}\right|$ and $\mu_{i_{1}}>0$.

Proposition 1. There exists a $h_{1} \in \mathbb{R}$ such that the Yukawa potential $V(x)$ is of negative curvature at all energies $h>h_{1}$.

Proof. Clearly the series defining $V$ and its derivatives are uniformly absolutely convergent on every compact $A \subset \mathbb{C}$ with $A \cap \mathscr{S}=\emptyset$ (no singularity points in $A$ ).

$$
\Delta V(x)=\sum_{I}\left(\left(\mu_{i_{1}}+r_{I}^{-1}\right)^{2}-\mu_{i_{1}} r_{I}^{-1}\right) V_{I}(x),
$$

with

$$
\begin{gathered}
V_{I}(x):=c_{i_{1}} \frac{\exp \left(-\mu_{i_{1}} r_{I}\right)}{r_{I}}, \\
(\nabla V(x))^{2}=\sum_{I} \sum_{J}\left(\mu_{i_{1}}+r_{I}^{-1}\right)\left(\mu_{j_{1}}+r_{J}^{-1}\right) \cos \left(\phi_{I}-\phi_{J}\right) V_{I}(x) V_{J}(x)
\end{gathered}
$$

with the multiindex $J=\left(j_{1}, j_{2}, j_{3}\right)$ and the angle $\phi_{I}$ between $x-x_{I}$ and, say, the $l_{1}$-direction.

Without loss of generality we may assume that the $s_{i_{1}}$ are interior points of the fundamental domain $\mathscr{D}$. Now

with

$$
\begin{aligned}
N(x):= & (h-V(x)) \Delta V(x)+(\nabla V(x))^{2}<h \Delta V(x) \\
& +\sum_{I} \sum_{J}\left(\left(\mu_{i_{1}}+r_{I}^{-1}\right)\left(\mu_{j_{1}}+r_{J}^{-1}-\mu_{i_{1}}-r_{I}^{-1}\right)+\mu_{i_{1}} r_{I}^{-1}\right) \times V_{I}(x) V_{J}(x) \\
= & h \Delta V(x)+\sum_{I=\left(i_{1}, 0,0\right)} \mu_{i_{1}} r_{I}^{-1} V_{I}^{2}(x)+A_{1}(x),
\end{aligned}
$$

$$
A_{1}(x):=\sum_{\neq\left(\left(i_{1}, 0,0\right),\left(i_{1}, 0,0\right)\right)}\left(\left(\mu_{i_{1}}+r_{i}^{-1}\right)\left(\mu_{j_{1}}+r_{J}^{-1}-\mu_{i_{1}}-r_{I}^{-1}\right)+\mu_{i_{1}} r_{I}^{-1}\right) \times V_{I}(x) V_{J}(x) .
$$

For $h>0$ the denominator in the expression for the curvature is bounded away from zero and for the numerator $N$ the inequality

$$
N<A_{1}+A_{2}+A_{3},
$$


with

$$
A_{2}(x):=h \sum_{I} \mu_{i_{1}}\left(\mu_{i_{1}}+r_{I}^{-1}\right) V_{I}(x)
$$

and

$$
A_{3}(x):=\sum_{I=\left(i_{1}, 0,0\right)} \frac{V_{I}(x)\left(\mu_{i_{1}} V_{I}(x)+h / r_{I}\right)}{r_{I}}
$$

is valid.

But there exists some $h_{2}$ such that for all $h>h_{2}>0, A_{1}+A_{2}<0$ everywhere. Indeed the leading order of the singularity in both terms is $r_{I}^{-2}$, with negative coefficients in $A_{2} / h$, and $A_{2} / h$ is strictly negative in the fundamental domain $\mathscr{D}$.

For $h>h_{3}:=\max _{i}\left(\left|\mu_{i} c_{i}\right|\right) A_{3}$ is negative definite. So for all $h>\max \left(h_{2}, h_{3}\right)$ the statement of Proposition 1 is true.

The condition $h_{1}>0$ could be expected and is necessary since the effective potential

$$
\frac{M^{2}}{2 r^{2}}+c \frac{\exp (-\mu r)}{r}
$$

of a single Yukawa potential can have minima greater than zero.

\subsection{Example 2: Potentials of Finite Ranges}

We use the same multiindex notation as above. Consider the function

$$
V: M_{1}^{\prime} \rightarrow \mathbb{R}, \quad V(x):=\sum_{I} V_{i_{1}}\left(r_{I}\right),
$$

with

$$
V_{i_{1}}(r):=-\frac{g_{i_{1}}(r)}{r}
$$

and $g_{i}: \mathbb{R}^{+} \rightarrow \mathbb{R}^{+}$being $C^{6}, g_{i}(0)>0, g_{i}(r)=0$ for all $r \geqq r_{i}$.

If one assumes that the supports of the $V_{i_{1}}\left(r_{I}\right)$ are disjoint, one obtains:

$$
(h-V(x)) \Delta V(x)+(\nabla V(x))^{2}=\sum_{I}\left(\left(h-V_{i_{1}}\left(r_{I}\right)\right) \Delta V_{i_{1}}\left(r_{I}\right)+\left(\nabla V_{i_{1}}\left(r_{I}\right)\right)^{2}\right) .
$$

So to show negative curvature of $V$ it is sufficient to show negative curvature of the $V_{i}$. Consider the following concrete example:

Proposition 2. With the above notation, let $g_{i}(r)$ be

$$
g_{i}(r):= \begin{cases}g_{i} \cos ^{7}\left(\lambda_{i} r\right), g_{i}>0 & r<\frac{\pi}{2 \lambda_{i}} \\ 0 & r \geqq \frac{\pi}{2 \lambda_{i}} .\end{cases}
$$

Then there exists a $h_{1}$ such that $V(x)$ is of negative curvature at all $h>h_{1}$, provided there exists no straight line in $\mathbb{C} \backslash \operatorname{supp}(V)$. 
Proof.

$$
\begin{gathered}
\Delta V_{i}(r)=\left(\frac{\partial^{2}}{\partial r^{2}}+\frac{1}{r} \frac{\partial}{\partial r}\right) V_{i}(r)=\frac{-r^{2} g_{i}^{\prime \prime}(r)+r g_{i}^{\prime}(r)-g_{i}(r)}{r^{3}} \\
\left(\nabla V_{i}(r)\right)^{2}=\frac{r^{2} g_{i}^{\prime 2}(r)-2 r g_{i}(r) g_{i}^{\prime}(r)+g_{i}^{2}(r)}{r^{4}}
\end{gathered}
$$

So the condition of negative curvature can be written as

$$
\left(-r^{2} g_{i}^{\prime \prime}(r)+r g_{i}^{\prime}(r)-g_{i}(r)\right) h \leqq g_{i}(r) g_{i}^{\prime}(r)+r\left(g_{i}(r) g_{i}^{\prime \prime}(r)-g_{i}^{\prime 2}(r)\right) \quad \text { for } \quad r<\frac{\pi}{2 \lambda_{i}}
$$

By rescaling this reduces to

$$
F(r) \cdot h \geqq 7 g_{i} \lambda_{i}^{2} \cos ^{7} r(\cos r \sin r+1) \quad \text { for } \quad r \in\left[0, \frac{\pi}{2}[\right.
$$

and

$$
F(r):=7 r^{2}\left(6 \sin ^{2} r-\cos ^{2} r\right)+7 r \cos r \sin r+\cos ^{2} r .
$$

By using the inequalities $\sin r \leqq r<\frac{\pi}{2} \sin r$, valid in the interval $\left[0, \frac{\pi}{2}[\right.$, one obtains:

$$
\begin{aligned}
F(r)= & 35 r^{2} \sin ^{2} r+\cos ^{2} r+7 r \cos r(\sin r-r \cos 2 r) \\
\geqq & 35 \sin ^{4} r+1-\sin ^{2} r+7 r \cos r \sin r\left(1-\frac{\pi}{2}\right) \\
\geqq & 35 \sin ^{4} r+1+\left(\frac{7 \pi}{2}\left(1-\frac{\pi}{2}\right)-1\right) \sin ^{2} r \\
= & \left(\sqrt{35} \sin ^{2} r-1\right)^{2}+\left(2 \sqrt{35}+\frac{7 \pi}{2}\left(1-\frac{\pi}{2}\right)-1\right) \sin ^{2} r \\
& >c>0
\end{aligned}
$$

for $r \in\left[0, \frac{\pi}{2}[\right.$.

Therefore there exists a $h_{1}>0$ such that for all $h>h_{1}$ the curvature of $V(x)$ is negative at $h$.

From simple geometric considerations it follows that one only needs to check nonexistence of straight lines for finitely many directions $d$ of the form $d=n_{1} l_{1}+n_{2} l_{2}, n_{1}, n_{2} \in \mathbb{Z}$.

\section{Diffusion on the Plane}

In the last sections the motion of a particle on the fundamental domain was considered. Now the motion on the universal covering space, i.e. the whole plane will be studied. It is proven that the motion is a kind of diffusion process.

This fact does not follow directly from the ergodic properties of the flow $\Phi_{X}$ in the compact manifold $X$. The compact surfaces of constant negative curvature, whose covering surface is the Lobatchewski plane, may serve as a counterexample, since the geodesic motion on the plane is not diffusive.

Clearly diffusion takes only place "in the large," since locally the flow may be linearised. 
Let $Y$ be the (noncompact) completion of $T_{1} M_{1}^{\prime}$, locally regularized as $X$ (see the remark after Theorem 2 for its definition). $\Phi_{Y}: \mathbb{R} \times Y \rightarrow Y$ denotes the flow in $Y$, and $\pi_{Y}: Y \rightarrow \mathbb{C}$ the projection onto the configuration space.

Let $\mu_{Y}$ denote the natural volume measure on $Y$, locally proportional to the product of the measure on $M_{1}^{\prime}$ defined by the metric $g_{1}^{\prime}$, and Lebesgue measure on $S^{1}$ (measure theoretically the points over the singularity set $\mathscr{S}$ are unimportant). The normalisation $\mu_{Y}\left(\pi_{Y}^{-1}(\mathscr{D})\right)=1$ is chosen. $\mu_{Y}$ is invariant with respect to the flow: $\Phi_{Y}^{*}\left(\mu_{Y}\right)=\mu_{Y}$.

We ask ourselves how a normalized $\left(\mu_{A}(Y)=1\right)$ distribution $\mu_{A}$ of initial conditions is spread over the lattice after a long time. Since we are only interested in typical initial conditions, we demand $\mu_{A}$ to be absolutely continuous relative to $\mu_{Y}$. By this we exclude e.g. the case of a $\mu_{A}$ concentrated on periodic orbits, where clearly no diffusion would take place. For convenience of the argument we demand the support of $\mu_{A}$ to be contained in $\pi_{Y}^{-1}(\mathscr{D})$, i.e. the particle to start in the fundamental domain, and we write $\mu_{A}$ for $\pi_{Y X *}\left(\mu_{A}\right)$, too. Observe that we can easily deal with the more general case of a $\mu_{A}$ whose support is bounded in $Y$ instead of being contained in $\pi_{Y}^{-1}(\mathscr{D})$, since then there exists a $k \in \mathbb{N}$ such that the support of $\mu_{A}$ projects to a subset of the enlarged fundamental domain

$$
\left\{c \in \mathbb{C} \mid c=i_{1} l_{1}+i_{2} l_{2}, i_{1}, i_{2} \in[-k, k[\} .\right.
$$

The position of a point $y \in Y$ in the plane can be uniquely expressed with the help of the lattice vectors $l_{1}, l_{2}$ by

$$
\pi_{Y}(y)=c_{1}(y) l_{1}+c_{2}(y) l_{2}
$$

with lattice coordinates $c_{1}, c_{2}: Y \rightarrow \mathbb{R}$.

For $\left(n_{1}, n_{2}\right) \in \mathbb{Z}^{2} \backslash\{(0,0)\}$ let $f: Y \rightarrow \mathbb{R}$ be defined by

$$
f(y):=n_{1} c_{1}(y)+n_{2} c_{2}(y) .
$$

If $a<b$ the set $\{y \in Y \mid a \leqq f(y) \leqq b\}$ lies over a strip in the configuration plane.

Theorem 4. There exists $a \sigma=\sigma\left(n_{1}, n_{2}\right)>0$ such that for all $a \leqq b$

$$
\lim _{t \rightarrow \infty} \mu_{A}\left(\left\{y \in Y \mid a \leqq \frac{f\left(\Phi_{Y}(t, y)\right)}{\sqrt{t}} \leqq b\right\}\right)=\frac{1}{\sigma \sqrt{2 \pi}} \int_{a}^{b} e^{-\left(\frac{u}{\sigma}\right)^{2}} d u .
$$

Proof. We know from Theorem 2 that the flow $\Phi_{X}$ in the compact manifold $X$ with natural volume $\mu_{X}$ is Anosov and $\mathbb{K}$. For flows of this type Ratner [24] has shown that a large class of functions in $L^{2}\left(X, \mu_{X}\right)$ satisfies the Central Limit Theorem. So we shall transform the statement of the theorem concerning $Y$ to a statement about $X$. To be specific, we shall consider the special representation $\Phi_{s}$ of $\Phi_{X}$ on an isomorphic model of $X$, constructed in $[23,24]$.

Consider the above function $f$ on $Y$. We write

$$
f\left(\Phi_{Y}(t, y)\right)=f(y)+\int_{0}^{t} g\left(\Phi_{X}\left(s, \pi_{Y X}(y)\right)\right) d s
$$

with $g: X \rightarrow \mathbb{R}$ unambiguously defined by

$$
g\left(\pi_{Y X}(y)\right):=\frac{\partial f\left(\Phi_{Y}(t, y)\right)}{\partial t} .
$$


So it is sufficient to prove that for all $a<b$,

$$
\lim _{t \rightarrow \infty} \mu_{A}\left(\left\{x \in X \mid a \leqq \frac{\int_{0}^{t} g\left(\Phi_{X}(t, x)\right) d t}{\sigma \sqrt{t}} \leqq b\right\}\right)=\frac{1}{\sqrt{2 \pi}} \int_{a}^{b} e^{-v^{2}} d v .
$$

Let $M(X)$ denote the space of Borel probability measures on $X$ equipped with the weak* topology. Then the flow $\Phi_{X}$ on $X$ induces an associated flow

$$
\tilde{\Phi}_{X}: \mathbb{R} \times M(X) \rightarrow M(X)
$$

on $M(X)$, given by

$$
\widetilde{\Phi}_{X}(t, \mu)(B):=\mu\left(\Phi_{X}(-t, B)\right)
$$

for all measurable sets $B C X$.

Since on $X$ the probability measure $\mu_{A}$ is absolutely continuous with respect to $\mu_{X}$, and $\mu_{X}$ is a strongly mixing measure, by Theorem 6.12 (ii) of [31],

$$
\lim _{t \rightarrow \infty} \widetilde{\Phi}_{X}\left(t, \mu_{A}\right)=\mu_{X}
$$

So it suffices to show that $g$ on $X$ satisfies the Central Limit Theorem (CLT) relative to the measure $\mu_{X}$.

In [23] Ratner showed for Anosov flows on a manifold $X$ the existence of a Markov partition $v$ of $X$ into parallelepipeds. Denoting the system of lower faces of this partition (i.e. the faces through which the flow enters the parallelepipeds) by $\mathscr{B}(v)$ and the time interval needed to traverse the parallelepiped starting at $x$ in its lower face by $l(x)$, she showed that $l(x)$ satisfies a Hölder condition on every continuity component of $\mathscr{B}(v)$. A mapping $T_{X}$ on the set-theoretic union $M_{X} \subset X$ of the lower faces is defined by the flow $\Phi_{X}$ :

$$
T_{X}: M_{X} \rightarrow M_{X} \quad x \mapsto \Phi_{X}(l(x), x) .
$$

Using the $r \times r$ transition matrix $A$ defined by the Markov partition of $X$, one defines the symbolic dynamical system $\left(\Sigma_{A}, \sigma\right)$ in the following way: Let $\Sigma:=\{1,2, \ldots, r\}^{\mathbb{Z}}$ be the space of doubly infinite sequences with metric

$$
d(\underline{x}, \underline{y}):=\sum_{i \in \mathbb{Z}} 2^{-|i|} \cdot\left(1-\delta_{x_{i} y_{i}}\right) .
$$

Then $\Sigma_{A} C \Sigma$ is defined as the (compact) subspace of bi-infinite sequences $\underline{x}$ with $A_{x_{\imath} x_{t+1}}=1$ for all $i \in \mathbb{Z}$ and $\sigma: \Sigma_{A} \rightarrow \Sigma_{A},(\sigma(\underline{x}))_{n}=x_{n+1}$ the shift.

There exists a continuous surjection $\varrho: \Sigma_{A} \rightarrow M_{X}$ such that $\varrho \circ \sigma(\underline{x})=T_{X} \circ \varrho(\underline{x})$. So for every $f: M_{X} \rightarrow \mathbb{R}$ one has a $\hat{f}: \Sigma_{A} \rightarrow \mathbb{R}$ given by $\hat{f}(\underline{x}):=f(\varrho(\underline{x}))$.

The special representation is then given by the special flow $\Phi_{s}: \mathbb{R} \times W \rightarrow W$ on

$$
W:=\left\{(\underline{x}, y) \in \Sigma_{A} \times \mathbb{R} \mid 0 \leqq y \leqq \hat{l}(\underline{x}),(\underline{x}, \hat{l}(\underline{x}))=(\sigma(\underline{x}), 0)\right\}
$$

by

$$
\Phi_{s}(t,(\underline{x}, y)):= \begin{cases}(\underline{x}, y+t) & 0 \leqq t<\hat{l}(\underline{x})-y \\ (\sigma(\underline{x}), y+t-\hat{l}(\underline{x})) & \widehat{l}(\underline{x})-y \leqq t<\inf _{\underline{x}} \hat{l}(\underline{x})\end{cases}
$$


and extension of this definition by using the group property $\Phi_{s}\left(t_{1}, \Phi_{s}\left(t_{2}, z\right)\right)$ $=\Phi_{s}\left(t_{1}+t_{2}, z\right)$ of the flow.

There exists a continuous map $\psi: W \rightarrow X$ such that

$$
\psi\left(\Phi_{s}(t, z)\right)=\Phi_{X}(t, \psi(z)) .
$$

Let $\mu_{W}$ be the $\Phi_{s}$-invariant Gibbs measure on $W$ which makes the flow $\Phi_{s}$ on $\left(W, \mu_{W}\right)$ isomorphic to $\Phi_{X}$ on $\left(X, \mu_{X}\right)\left(\mu_{X}\right.$ was defined as the normalized natural volume on $X$ ). Then $\mu_{X}$ induces a $\sigma$-invariant measure $\mu_{\Sigma_{A}}$ on $\Sigma_{A}$ :

$$
d \mu_{W}=\frac{d \mu_{\Sigma_{A}} \cdot d t}{\bar{l}},
$$

with a normalization constant $\bar{l}$.

The expectation $E(\hat{f})$ of a function $\hat{f}: \Sigma_{A} \rightarrow \mathbb{R}$ is defined by

So $\bar{l}$ equals $E(\hat{l})$.

$$
E(\hat{f}):=\int_{\Sigma_{A}} \hat{f}(\underline{x}) d \mu_{\Sigma_{A}} .
$$

Since $\left(X, \mu_{X}\right)$ and $\left(W, \mu_{W}\right)$ are isomorphic, it suffices to show the CLT for

$$
\hat{\mathrm{g}}: W \rightarrow \mathbb{R}, \quad \hat{g}(x):=g(\psi(x)) .
$$

$\hat{g}$ is continuous since $g$ is even differentiable.

Theorem 2.1 of [24] says that such a continuous function $\hat{g}: W \rightarrow \mathbb{R}$ satisfies the CLT if the homology equation

$$
\hat{V} \hat{h}=\hat{g}-\overline{\mathrm{g}}
$$

has no solution $\hat{h} \in L^{2}\left(W, \mu_{W}\right)$. Here $\hat{V}$ is the generator of the flow in $L^{2}\left(W, \mu_{W}\right)$ adjoint to the flow $\Phi_{W} \cdot \overline{\mathrm{g}}:=\int_{W} \hat{g}(w) d \mu_{W}$ is the mean value of $\hat{\mathrm{g}}$ and

$$
\overline{\hat{g}}=\int_{X} g(x) d \mu_{X}=\int_{M_{2}^{\prime}} \int_{S^{1}} g(x) d s d M_{2}^{\prime}=0
$$

by integration over the fibre $S^{1}$ parametrizing the direction of the particle.

Assume that there exists such a $\hat{h}$. Using a similar argument as in Bunimovich and Sinai [9] we will show that under this condition the flow of a manifold covering $X$ cannot be ergodic.

Since by [23] the set of points $x \in X$ where $\psi^{-1}: X \rightarrow W$ is not well defined has Lebesgue measure 0 , there exists a $h: X \rightarrow \mathbb{R}$ with $\psi(h)=h$ a.e. which solves the corresponding homology equation for $g: X \rightarrow \mathbb{R}$ almost everywhere.

By integration one gets

$$
\int_{0}^{t} g\left(\Phi_{X}(s, x)\right) d s=h\left(\Phi_{X}(t, x)\right)-h(x)
$$

almost everywhere.

So for all measurable sets $\widetilde{A} \subset \operatorname{supp}\left(\mu_{A}\right) \subset Y$ with $\mu_{A}(\tilde{A})>0$ and all $\varepsilon>0$, one finds a $C>0$ such that for all $t \in \mathbb{R}$,

$$
\mu_{Y}\left\{x \in X|| \int_{0}^{t} g\left(\Phi_{X}(s, x)\right) d s \mid>C\right\}<\varepsilon \cdot \mu_{Y}(\tilde{A}) .
$$


Let the three dimensional compact manifold $X_{k}$ be the completion of $T_{1}\left(M_{1}^{\prime} / k \cdot \mathscr{C}\right)$, $k \in \mathbb{N}$, as in Theorem 2 and $\pi_{Y X_{k}}: Y \rightarrow X_{k}$ the induced covering. So $X_{k}$ covers $X$ $k \times k$ times:

$$
\pi_{X_{k} X}: X_{k} \rightarrow X, \quad \pi_{X_{k} X}(x):=\pi_{Y X} \circ \pi_{Y X_{k}}^{-1}(x) .
$$

Let $\Phi_{X_{k}}: \mathbb{R} \times X_{k} \rightarrow X_{k}$ be the pullback of the flow $\Phi_{X}$ and $\mu_{X_{k}}$ the normalized natural volume on $X_{k}$.

Consider the set $S:=\left\{y \in Y \mid d_{1}<f(y)<d_{2}\right\}$ with

$$
d_{1}:=\sup _{y \in \operatorname{supp}\left(\mu_{A}\right)} f(y)+C, \quad d_{2}:=\inf _{y \in \operatorname{supp}\left(\mu_{A}\right)} f(y)-C .
$$

Since $f$ was defined as an integer combination of the lattice coordinates $c_{1}$ and $c_{2}$, there exists a $k \in \mathbb{N}$ such that $\mu_{X_{k}}\left(S_{k}\right)<\frac{1}{2}$ for $S_{k}:=\pi_{Y X_{k}}(S)$.

Using the sets $B_{k}:=X_{k} \backslash S_{k}$ and $A_{k}:=\pi_{Y X_{k}} \tilde{A} \subset X_{k}$, for all $t_{0} \in \mathbb{R}$ one gets

$$
\lim _{n \rightarrow \infty} \frac{1}{n} \sum_{i=0}^{n-1} \mu_{X_{k}}\left(\Phi_{X_{k}}\left(n t_{0}, A_{k}\right) \cap B_{k}\right)<\varepsilon \mu_{X_{k}}\left(A_{k}\right)<\mu_{X_{k}}\left(A_{k}\right) \mu_{X_{k}}\left(B_{k}\right)
$$

for small enough $\varepsilon>0$, which means that $\Phi_{X_{k}}$ is not ergodic.

But by Theorem 3 the flow $\Phi_{X_{k}}$ is ergodic. So we have derived a contradiction and thus we have shown that no solution of the homology equation exists.

Remark. Observe that we have not shown that the $2 \times 2$ covariance matrix of the diffusion process is nondegenerate. There could exist an eigenvector with eigenvalue 0 pointing in a direction which is irrational relative to the lattice $\mathscr{C}$.

In [9] Bunimovich and Sinai showed that the motion of a billard in a plane with periodic convex obstacles is a diffusion process with nondegenerate covariance. It is not clear to me whether their proof of nondegeneracy carries over to the above situation, since they published only a short sketch of the argument.

\section{Topological Properties of the Flow}

By Theorem 3 and Ornstein and Weiss [22] all coulombic potentials of negative curvature lead to measure theoretically isomorphic flows (neglecting a reparametrization of the time variable).

Now we want to show that all coulombic potentials with the same number $n$ of singularities and negative curvature at some energy $h$ have topologically conjugate flows.

To do so we will use the existence of a simple path in the space $\mathscr{C}$ of metrics of negative curvature on a given surface $\mathscr{F}$, which are conformally flat for a fixed atlas $\mathscr{A}$.

Lemma 2. $\mathscr{C}$ is convex. The subspace of metrics having strictly negative curvature is convex, too.

Proof. By assumption the two metrics $g^{\mathrm{I}}, g^{\mathrm{II}} \in \mathscr{C}$ have the local form

$$
g_{i k}^{\mathrm{I}}(x)=f^{\mathrm{I}}(x) \delta_{i k} \text { and } g_{i k}^{\mathrm{II}}(x)=f^{\mathrm{II}}(x) \delta_{i k}
$$


in some chart of $\mathscr{A}$. Defining the convex combination

$$
g_{i k}^{\lambda}(x):=f^{\lambda}(x) \delta_{i k}:=\left(\lambda f^{\mathrm{I}}(x)+(1-\lambda) f^{\mathrm{II}}(x)\right) \delta_{i k}
$$

with curvature

$$
K^{\lambda}(x)=\frac{f^{\lambda}(x) \Delta f^{\lambda}(x)+\left(\nabla f^{\lambda}(x)\right)^{2}}{\left(f^{\lambda}(x)\right)^{3}}
$$

we have to show that for all $\lambda \in[0,1]$ the nominator is $\leqq 0$.

$$
\begin{aligned}
f^{\lambda}(x) \Delta f^{\lambda}(x)+\left(\nabla f^{\lambda}(x)\right)^{2}= & \lambda^{2}\left(f^{\mathrm{I}}(x) \Delta f^{\mathrm{I}}(x)+\left(\nabla f^{\mathrm{I}}(x)\right)^{2}\right) \\
& +(1-\lambda)^{2}\left(f^{\mathrm{I}}(x) \Delta f^{\mathrm{II}}(x)+\left(\nabla f^{\mathrm{II}}(x)\right)^{2}\right) \\
& +\lambda(1-\lambda)\left(f^{\mathrm{I}}(x) \Delta f^{\mathrm{II}}(x)+f^{\mathrm{II}}(x) \Delta f^{\mathrm{I}}(x)\right. \\
& \left.+2 \nabla f^{\mathrm{I}}(x) \cdot \nabla f^{\mathrm{II}}(x)\right) \\
\leqq & \lambda(1-\lambda)\left(f^{\mathrm{I}}(x) \Delta f^{\mathrm{II}}(x)+f^{\mathrm{II}}(x) \Delta f^{\mathrm{I}}(x)\right. \\
& \left.+2 \nabla f^{\mathrm{I}}(x) \cdot \nabla f^{\mathrm{II}}(x)\right) \\
\leqq & -\lambda(1-\lambda)\left(\sqrt{f^{\mathrm{I}}(x)\left|\Delta f^{\mathrm{II}}(x)\right|}-\sqrt{f^{\mathrm{II}}(x)\left|\Delta f^{\mathrm{I}}(x)\right|}\right)^{2} \\
\leqq & 0 .
\end{aligned}
$$

The first inequality is strict in the case of strictly negative curvature.

Theorem 5. Let $V^{A}$ and $V^{B}$ be coulombic potentials with the same number $n$ of singularities, which are of negative curvature at energies $h^{A}$ respectively $h^{B}$. With $X^{A}$ respectively $X^{B}$ denoting the space $X$ of the two systems, the flows on $X^{A}$ and $X^{B}$ are topologically conjugate, i.e. there exists a homeomorphism $\mathscr{H}: X^{A} \rightarrow X^{B}$ carrying oriented orbits to oriented orbits.

Proof. Two arbitrary coulombic potentials with $n$ singularities lead to diffeomorphic differentiable manifolds $M_{4}$. So we consider different coulombic potentials $V^{(\lambda)}(x)$ as leading to different metrics $g_{4}^{(\lambda)}$ on a fixed manifold $M_{4}$.

Now we construct a path in the space $\mathscr{P}_{n}$ of coulombic potentials with $n$ singularities between $V^{A}$ and $V^{B}$ :

$$
\begin{aligned}
& \gamma:[1,5] \rightarrow \mathscr{P}_{n}, \lambda \mapsto V^{(\lambda)}, \\
& V^{(1)}:=V^{A}, V^{(5)}:=V^{B} .
\end{aligned}
$$

This path will in turn lead to a continuous path in the space of metrics $g_{4}^{(\lambda)}$ on $M_{4}$ entirely contained in the subspace $\mathscr{C}$ of metrics of negative curvature, given an energy function $h:[1,5] \rightarrow \mathbb{R}$.

Without loss of generality we choose the lattice vectors $l_{i}^{A}\left(l_{i}^{B}\right)$ generating the lattice $\mathscr{C}^{A}\left(\mathscr{C}^{B}\right)$ of the potential $V^{A}\left(V^{B}\right)$ such that the imaginary parts $\Im\left(l l_{1}^{A}\right)$ $=\mathfrak{J}\left(l_{1}^{B}\right)=0$. Let $s_{i}^{A}\left(s_{i}^{B}\right)$ denote the points of singularity of $V^{A}\left(V^{B}\right)$.

To construct $\gamma$ it is convenient to work with potentials of finite range $R>\frac{1}{2} \max \left(\left|l_{1}^{A}\right|,\left|l_{2}^{A}\right|,\left|l_{1}^{B}\right|,\left|l_{2}^{B}\right|\right)$. If we place such potentials on a sublattice generated by $m_{1} l_{1}^{A}$ and $m_{2} l_{2}^{A}$ with $m_{1}>\frac{2 R}{\left|l_{1}^{A}\right|}, m_{2}>\frac{2 R}{\left|\mathfrak{I}\left(l_{2}^{A}\right)\right|}$, their supports do not overlap. 


\section{Defining}

$$
U(r):=\left\{\begin{array}{ll}
-\frac{\cos ^{7}\left(\frac{\pi r}{2 R}\right)}{r} & r<R \\
0 & r \geqq R
\end{array},\right.
$$

we know from Proposition 2 that there exists a $h_{U}>0$ such that the metric $g_{i k}(x):=\left(h_{U}-U(|x|)\right) \delta_{i k}$ leads to strictly negative curvature for $|x|<R$. By iterated use of Lemma 2 one sees that

$$
V^{(2)}(x):=\sum_{i=1}^{n} \sum_{n_{1}, n_{2} \in \mathbb{Z}} U\left(\left|x-s_{i}^{A}-n_{1} l_{1}^{A}-n_{2} l_{2}^{A}\right|\right)
$$

is of strictly negative curvature at energy $h(2):=m_{1} m_{2} n h_{U}$, since the support $\operatorname{supp}\left(V^{(2)}\right)$ equals $M_{1}^{\prime A}:=\mathbb{C} \backslash\left\{s_{i}^{A}+\mathscr{C}^{A}\right\}$.

$$
V^{(\lambda)}(x):=(2-\lambda) V^{(1)}(x)+(\lambda-1) V^{(2)}(x)
$$

interpolates between $V^{(1)}$ and $V^{(2)}$ and is of strictly negative curvature at energy $h(\lambda):=(2-\lambda) h^{A}+(\lambda-1) h(2)$ for $1<\lambda \leqq 2$.

Similarly we define

$$
\begin{aligned}
V^{(4)}(x) & :=\sum_{i=1}^{n} \sum_{n_{1}, n_{2} \in \mathbb{Z}} U\left(\left|x-s_{i}^{B}-n_{1} l_{1}^{B}-n_{2} l_{2}^{B}\right|\right), \\
V^{(\lambda)}(x) & :=(5-\lambda) V^{(4)}(x)+(\lambda-4) V^{(5)}(x),
\end{aligned}
$$

and

$$
h(\lambda):=(5-\lambda) h(2)+(\lambda-4) h^{(B)} \quad \text { for } \quad \lambda \in[4,5] .
$$

In the interval $\lambda \in[2,3]$ we rearrange the lattice. Given the unique decomposition of $s_{i}^{A}=\mu_{i}^{A} l_{1}^{A}+v_{i}^{A} l_{2}^{A}$ we define

$$
\begin{gathered}
V^{(\lambda)}(x):=\sum_{i=1}^{n} \sum_{n_{1}, n_{2} \in \mathbb{Z}} U\left(\mid x-(3-\lambda)\left(\left(n_{1}+\mu_{i}^{A}\right) l_{1}^{A}+\left(n_{2}+v_{i}^{A}\right) l_{2}^{A}\right)\right. \\
\left.-(\lambda-2)\left(\left(n_{1}+\mu_{i}^{A}\right) l_{1}^{B}+\left(n_{2}+v_{i}^{A}\right) l_{2}^{B}\right) \mid\right) \\
h(\lambda):=h(2) \text { for } \lambda \in[2,3] .
\end{gathered}
$$

Now for some permutation $\chi:\{1, \ldots, n\} \rightarrow\{1, \ldots, n\}$ choose $n$ paths $\delta_{i}:[3,4] \rightarrow \mathbb{C}$ such that

$$
\begin{gathered}
\left(\delta_{i}(\lambda)-\delta_{j}(\lambda)\right) \bmod \mathscr{C}^{B} \neq 0 \quad \text { for all } \quad i \neq j \quad \text { and } \lambda \in[3,4] \\
\delta_{i}(3)=\mu_{i}^{A} l_{1}^{B}+v_{i}^{A} l_{2}^{B} \text { and } \delta_{i}(4)=s_{\chi(i)}^{B} .
\end{gathered}
$$

Define

$$
V^{(\lambda)}(x):=\sum_{i=1}^{n} \sum_{n_{1}, n_{2} \in \mathbb{Z}} U\left(\left|x-\delta_{i}(\lambda)-n_{1} l_{1}^{B}-n_{2} l_{2}^{B}\right|\right)
$$

and $h(\lambda):=h(2)$ for $\lambda \in[3,4]$. 
No singularities met during the whole deformation process. By (iterated) application of Lemma 2 one sees that the curvature $K(x)$ on $M_{4}$ is strictly negative for $1<\lambda<5$. So the geodesic flow on $T_{1} M_{4}$ is Anosov for all metrics $g_{4}^{(\lambda)}, \lambda \in[1,5]$. Since Anosov flows are structurally stable, the flows $\Phi_{4}^{A}$ and $\Phi_{4}^{B}$ on $T_{1} M_{4}$ corresponding to the potentials $V^{A}$ and $V^{B}$ and energies $h^{A}$ and $h^{B}$ must be topologically conjugate. By Theorem 2 there exists a family $\Phi_{X}^{(\lambda)}: \mathbb{R} \times X \rightarrow X$ of Anosov flows on $X, \lambda \in[1,5]$, and thus there exists the homeomorphism $\mathscr{H}: X^{A} \rightarrow X^{B}$ carrying oriented orbits to oriented orbits.

Remarks. 1. There exist not only $n !-1$ but an infinity of nontrivial selfconjugations of the flow $\Phi_{X}$ on $X$ for a coulombic potential with $n$ singularities, which is of negative curvature at energy $h(n>1)$. For besides permuting the singularities, one can choose the paths $\delta_{i}$ in the proof of the last theorem in many different ways. Even closed paths change the phase portrait. This is illustrated in Fig. 3. There $\delta_{1}$, $\delta_{2}$, and $\delta_{3}$ are constant (the position of the singularities $s_{1}, s_{2}$, and $s_{3}$ is not changed), and $s_{4}$ is moved around $s_{3}$ along the path $\delta_{4}$. Accordingly the form of a closed orbit enclosing $s_{1}, s_{2}$, and $s_{3}$ changes drastically.

2. The restriction to the same number of singularities in the statement of Theorem 5 was inessential. For let $V^{A}\left(V^{B}\right)$ have $n^{A}\left(n^{B}\right)$ singularities in their respective fundamental domains. One just has to enlarge both fundamental domains so that they contain $n^{A} \times n^{B}$ singularities. Then Theorem 5 applies.

Now we will use simple Morse theory (see, e.g. Milnor [20]) to obtain information about the periodic solutions.

The manifold $M_{2}^{\prime}$ is topologically the torus $M_{2}$ with $n$ points $\left\{s_{1}, \ldots, s_{n}\right\}$ deleted. The first homotopy group $\pi_{1}\left(M_{2}^{\prime}\right)$ is the free group on $n+1$ generators and it can be used to analyse the closed orbits.

Since $\left(M_{2}^{\prime}, g_{2}^{\prime}\right)$ is incomplete, one cannot apply Morse theory to it. But using Morse theory, one can analyse all closed geodesics on the complete Riemannian manifold $\left(M_{4}, g_{4}\right)$ and project them down using the covering $\tilde{\pi}_{42 *}: T_{1} M_{4} \rightarrow X$ established in Theorem 2.
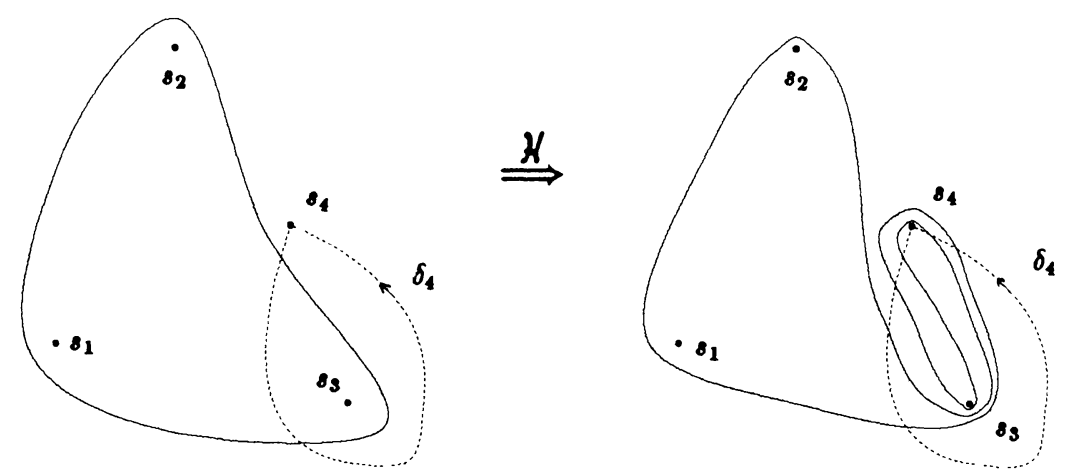

Fig. 3. A closed path in the space of coulombic potentials deforming one periodic orbit into another one 
Definition. A closed orbit $\gamma$ of a flow $\Phi_{M}: \mathbb{R} \times M \rightarrow M$ is a map

$$
\gamma:[0, T] \rightarrow M \quad \gamma(t):=\Phi_{M}(t, \gamma(0))
$$

with period $T>0$ and $\gamma(T)=\gamma(0)$.

Two closed orbits $\gamma_{1}, \gamma_{2}, \gamma_{i}:\left[0, T_{i}\right] \rightarrow M$ are called equivalent if $T_{1}=T_{2}$ and $\gamma_{1}\left(\left[0, T_{1}\right]\right)=\gamma_{2}\left(\left[0, T_{2}\right]\right)$. Clearly this defines an equivalence relation. The set of closed orbits of $\Phi_{M}$ modulo this equivalence relation is called the closed orbit set of $\Phi_{M}$.

Remark. So we identify all closed orbits which are related by the natural $S O(2)$ action, but we discern closed orbits whose periods are $n_{i} \cdot T_{\min }$ with $n_{1}, n_{2} \in \mathbb{N}$, $n_{1} \neq n_{2}$. Insofar this definition is nonstandard (e.g. in Abraham and Marsden a closed orbit is defined as the set $\gamma([0, T]) \subset M)$. We sometimes write $(x, T) \in M \times \mathbb{R}$ for an element of the closed orbit set with representative $\gamma:[0, T] \rightarrow M$ and $x=\gamma(0)$. So $\left(x_{1}, T_{1}\right) \sim\left(x_{2}, T_{2}\right)$ iff $x_{1}=\Phi\left(t, x_{2}\right)$ for some $t \in \mathbb{R}$ and $T_{1}=T_{2}$.

We want to analyse the closed orbit set $\mathbf{P}_{X}$ of $\Phi_{X}$ using the homotopy group $\pi_{1}\left(M_{2}^{\prime}\right)$. Since $\left(M_{2}^{\prime}, g_{2}^{\prime}\right)$ is incomplete, not in every homotopy class of $\pi_{1}\left(M_{2}^{\prime}\right) \backslash\{e\}$ there exists a closed orbit.

Let $y \in M_{4}$ project to $\pi_{42}(y)=: x \in M_{2}^{\prime}$ and let $\gamma:[0,1] \rightarrow M_{2}^{\prime}$ be a $x$-based loop. For the $y$-based curve $\varepsilon:[0,1] \rightarrow M_{4}^{\prime}$ projecting to $\gamma\left(\pi_{42}(\varepsilon(t))=\gamma(t), t \in[0,1]\right)$, there exists a group element $g \in G$ such that $\varepsilon(1)=\psi_{g}(\varepsilon(0))$ (see Sect. 2). Since $G=\mathbb{Z}_{2} \times \mathbb{Z}_{2} \times \mathbb{Z}_{2}, g \cdot g=e$ for all $g \in G$.

If $\varepsilon(1)=\varepsilon(0)$, let $l(\gamma):=1$, otherwise $l(\gamma):=2$.

Now we define a $y$-based loop $\delta:[0,1] \rightarrow M_{4}$, covering $\gamma$. If $l(\gamma)=1, \delta:=\varepsilon$. If $l(\gamma)=2$, we define $\delta$ by

$$
\delta(t):=\left(\psi_{g}(\varepsilon) * \varepsilon\right)(t),
$$

i.e. we concatenate $\varepsilon$ with the translated curve $\psi_{g}(\varepsilon)$.

Definition. $\delta$ is called a l-covering loop of $\gamma$. If $\delta$ is homotopic to the constant loop in $M_{4}$, then $\gamma$ is called $\pi_{42}$-trivial.

Every loop $\gamma_{2}:[0,1] \rightarrow M_{2}^{\prime}$ homotopic to a $\pi_{42}$-trivial loop $\gamma_{1}$ is $\pi_{42}$-trivial. So $\pi_{42}$-triviality is a property of homotopy classes.

Denote by $E \subset \pi_{1}\left(M_{2}^{\prime}, x\right)$ the set of homotopy classes whose representatives are $\pi_{42}$-trivial. As noted above, there exists an isomorphism

$$
i: \pi_{1}\left(M_{2}^{\prime}, x\right) \rightarrow F_{n+1}
$$

with $F_{n+1}$ the free group on $n+1$ generators. For simplicity we write $i(E)=: E$.

Theorem 6. Assume that the coulombic potential $V$ is of negative curvature at energy $h$. Then there exists a surjection

$$
\sigma: F_{n+1} \backslash_{E} \rightarrow \mathbf{P}_{X}
$$

with $F_{n+1}$ the free group on $n+1$ generators and $\mathbf{P}_{X}$ the closed orbit set of $\Phi_{X}$.

Proof. Let $\pi_{1}\left(M_{4}, y\right)$ denote the first homotopy group of $M_{4}$ with base point $y$ and let $y$ project to $\pi_{42}(y)=x \in M_{2}^{\prime}$. (Then $\pi_{1}\left(M_{4}, y\right)$ is generated by $y$-based loops $g_{1}, \ldots, g_{4 n+2}$ with the relation

$$
\left[g_{4 n+2}\right]^{-1}\left[g_{4 n+1}\right]^{-1}\left[g_{4 n+2}\right]\left[g_{4 n+1}\right] \ldots\left[g_{2}\right]^{-1}\left[g_{1}\right]^{-1}\left[g_{2}\right]\left[g_{1}\right]=e
$$

of their homotopy classes $\left[g_{1}\right], \ldots,\left[g_{4 n+2}\right]$, see, e.g. [19].) 
Let $\gamma:[0,1] \rightarrow M_{2}^{\prime}$ be a $x$-based loop with $[\gamma] \in \pi_{1}\left(M_{2}^{\prime}, x\right) \backslash_{E}$ and $\delta:[0,1] \rightarrow M_{4}$ its $y$-based $l$-covering loop. By definition of $E$ the homotopy class $[\delta] \in \pi_{1}\left(M_{4}, y\right)$ is nontrivial.

So we can define the map

$$
\tau: \pi_{1}\left(M_{2}^{\prime}, x\right) \backslash_{E} \rightarrow\left\{\pi_{1}\left(M_{4}, y\right) \backslash_{\{e\}}\right\} \times\{1,2\}, \quad \tau([\gamma]) \mapsto([\delta], l(\gamma)) .
$$

Morse theory tells us in case of smooth Riemannian manifolds $(M, g)$ for every nontrivial homotopy class $\kappa \in \pi_{1}(M)$ there exists a closed geodesic $\zeta:\left[0, T_{\zeta}\right] \rightarrow T_{1} M$ in the unit tangent bundle $\pi: T_{1} M \rightarrow M$ with $[\pi(\zeta)]=\kappa$.

In our case $\left(M_{4}, g_{4}\right)$ is of class $C^{6}$ by Theorem 1 . According to Theorems 8.46 and 8.47 of Schwartz [27, Chap. VIII], under this minimal differentiability condition the above statement is still true.

So there exists a closed geodesic $\zeta(\delta):\left[0, T_{\zeta}\right] \rightarrow T_{1} M_{4}$ in the unit tangent bundle $\pi_{4}: T_{1} M_{4} \rightarrow M_{4}$ with $\left[\pi_{4}(\zeta)\right]=[\delta]$. This geodesic is unique [up to selection of the initial point $\zeta(0)$ ], since the usual uniqueness proof for strictly negative curvature, using a Gauss-Bonnet argument (see, e.g. [16]), carries over to our situation.

So we have a well-defined map

$$
\varrho:\left\{\pi_{1}\left(M_{4}, y\right) \backslash\{e\}\right\} \times\{1,2\} \rightarrow \mathbf{P}_{4} \times\{1,2\}
$$

with

$$
\varrho([\delta], l):=\left(\left(\zeta(\delta)(0), T_{\zeta}\right), l\right),
$$

$\mathbf{P}_{4}$ being the closed orbit set of the geodesic flow $\Phi_{4}$ in $T_{1} M_{4}$, and $\left[\pi_{4}(\zeta)\right] \in \pi_{1}\left(M_{4}\right)$ the equivalence class of the geodesic $\zeta$.

By Theorem 2 the flow in $X$ is related to the geodesic flow in $T_{1} M_{4}$ by

$$
\Phi_{X}\left(t, \tilde{\pi}_{42 *}(z)\right)=\tilde{\pi}_{42 *} \circ \Phi_{4}(t, z) .
$$

Projecting the closed geodesic $\zeta:\left[0, T_{\zeta}\right] \rightarrow T_{1} M_{4}$ to the orbit $O:\left[0, T_{\zeta}\right] \rightarrow X$ with $O(t):=\tilde{\pi}_{42 *}(\zeta(t))$, one gets the result that every point $O(t) \in X$ is covered $l$ times by points in $\zeta\left(\left[0, T_{\zeta}\right]\right)$, since $\tilde{\pi}_{42 *}: T_{1} M_{4} \rightarrow X$ is an unbranched covering.

So on the image

$$
I:=\varrho \circ \tau\left(\pi_{1}\left(M_{2}^{\prime}, x\right) \backslash_{E}\right) \subset \mathbf{P}_{4} \times\{1,2\},
$$

the map

$$
\chi: I \rightarrow \mathbf{P}_{X} \quad \chi((z, T), l):=\left(\tilde{\pi}_{42 *}(z), T / l\right)
$$

is well defined [i.e. $\Phi_{X}\left(T / l, \tilde{\pi}_{42 *}(z)\right)=\tilde{\pi}_{42 *}(z)$ ], and we get a map

$$
\sigma: F_{n+1} \backslash_{E} \rightarrow \mathbf{P}_{X} \quad \sigma:=\chi \circ \varrho \circ \tau \circ i^{-1} .
$$

Every closed orbit in $X$, considered as a subset $\left(\cong S^{1}\right)$ of $X$, is a projection of a closed geodesic in $T_{1} M_{4}$. On the other hand every closed orbit in $\mathbf{P}_{4}$ is in the set

$$
\varrho \circ \tau\left(\pi_{1}\left(M_{2}^{\prime}, x\right) \backslash_{E}\right),
$$

disregarding the second factor of $\varrho$. So every closed orbit in $X$, considered as a subset of $X$, is in $\sigma\left(F_{n+1} \backslash_{E}\right)$. But also with respect to the period of the orbits $\sigma$ is surjective. 
Remarks. 1. Clearly the interesting point is not the mere existence of the surjection stated in the theorem but its explicit construction given in the proof. But note that $\sigma$ is not injective and that the relation between the homotopy classes of $M_{2}^{\prime}$ and the closed orbits in $X$ is of a rather indirect nature, due to the incompleteness of $\left(M_{2}^{\prime}, g_{2}^{\prime}\right)$. For example, all 2-collision orbits, whose homotopy classes in $M_{2}^{\prime}$ are undefined, are in $\mathbf{P}_{X}$.

2. The following assertions of Theorem 5.3.4 in [17] carry directly over to $X$ : - All closed orbits in $X$ are hyperbolic and have index 0.

- The set $\mathbf{P}_{X}$ of closed orbits, considered as a subset of $X$, is dense in $X$.

- The flow $\Phi_{X}$ on $X$ is transitive in the following sense: There exists a subset $\operatorname{Trans}(X)$ of full measure in $X$ such that for $x_{0} \in \operatorname{Trans}(X)$ the orbit $\Phi_{X}\left(t, x_{0}\right), t \geqq 0$ or $\leqq 0$ is dense in $X$.

- The number of closed orbits whose length is smaller than $x \in \mathbb{R}^{+}$grows asymptotically as $\exp (c x)$ with some positive constant $c$.

\section{Concluding Remarks}

All periodic potentials with negative singularities of type

$$
-r^{-2\left(1-\frac{1}{n}\right)}, n \in \mathbb{N} \backslash\{0,1\}
$$

can be analysed by analogous methods. One just uses branched coverings with local branch numbers $n-1$. Since

$$
\int_{0}^{a} r^{\frac{1}{n}-1} d r=n \cdot a^{\frac{1}{n}}
$$

the manifolds corresponding to $\left(M_{2}^{\prime}, g_{2}^{\prime}\right)$ in these cases are geodesically incomplete.

In the more physical case of $-r^{-2}$-singularities, representing for example a nonrelativistic black hole, something new happens. In these cases $\left(M_{2}^{\prime}, g_{2}^{\prime}\right)$ is geodesically complete.

As a consequence, one need not (and cannot) perform the branched covering construction. Then to every $g \in \pi_{1}\left(M_{2}^{\prime}\right), g \neq e$ corresponds a closed geodesic $\gamma$ with $[\gamma]=g$.

For several reasons it is not so easy to obtain equivalent information on the motion of a classical particle in a three dimensional potential with coulombic singularities.

The inapplicability of complex function theory itself does not seem to be so problematic, since one could replace Levi-Civita transformation by the Kustaanheimo-Stiefel transformation [30].

But the sectional curvature cannot have a negative upper bound in the three dimensional case, for one may easily construct examples of closed geodesics of nontrivial index.

The second problem is that in three dimensions the first homotopy group does not see the singularities but only the torus periodicity: $\pi_{1} \cong \mathbb{Z}^{3}$. One may use higher homotopy groups, but it is clear that they cannot provide as many closed geodesics as in the 2-dimensional case, since they are abelian. 
Concerning the related quantum problem, one may use the closed geodesics as a starting point for semiclassical calculations, as has been done by Berry ([7] and references therein), Gutzwiller [15] and others. In this case, too, the covering space may be of some use. For example, the spectrum of $\Delta+\frac{1}{r}$ is related to the harmonic oscillator in two dimensions by the Levi-Civita transformation.

Acknowledgement. I thank my thesis advisor Prof. Dr. R. Schrader for suggestions and support of this work.

\section{References}

1. Abraham, R., Marsden, J.E.: Foundations of mechanics. Reading: The Benjamin/Cummings 1978

2. Anosov, D.V.: Geodesic flows on closed manifolds with negative curvature. Proc. of the Steklov Institute of Mathematics, Vol. 90, Providence: Am. Math. Soc. (1969

3. Arnold, V.I.: Mathematical methods of classical mechanics. Graduate Texts in Mathematics, Vol. 60. Berlin, Heidelberg, New York: Springer 1978

4. Arnold, V.I.: Geometrical methods in the theory of ordinary differential equations. Grundlehren der mathematischen Wissenschaften, Vol. 250. Berlin, Heidelberg, New York: Springer 1983

5. Arnold, V.I., Avez, A.: Ergodic problems of classical mechanics. New York: W. A. Benjamin 1968

6. Behnke, H., Sommer, F.: Theorie der analytischen Funktionen einer komplexen Variablen. Berlin, Heidelberg, New York: Springer 1983

7. Berry, M.: Semiclassical mechanics of regular and irregular motion. Les Houches Session XXXV; Amsterdam, New York, Oxford: North-Holland 1981

8. Brown, J.R.: Ergodic theory and topological dynamics. New York: Academic Press 1976

9. Bunimovich, L.A., Sinai, Ya.G.: Statistical properties of Lorentz gas with periodic configuration of scatterers. Commun. Math. Phys. 78, 479-497 (1981)

10. Easton, R.: Regularisation of vector fields by surgery. J. Differ. Equations 10, 92-99 (1971)

11. Efimov, N.V.: Impossibility of a complete regular surface in Euclidean 3-space whose Gaussian curvature has a negative upper bound. Sov. Math. 4, 843-846 (1963)

12. Farkas, H.M., Kra, I.: Riemann surfaces. Graduate Texts in Mathematics, Vol. 71. Berlin, Heidelberg, New York: Springer 1977

13. Gallavotti, G.: Lectures on the billard. In: Dynamical systems, theory and applications. Moser, J. (ed.). Lecture Notes in Physics, Vol. 38. Berlin, Heidelberg, New York: Springer 1974

14. Gallavotti, G., Ornstein, D.S.: Billards and Bernoulli schemes. Commun. Math. Phys. 38, 83-101 (1974)

15. Gutzwiller, M.C.: The quantization of a classically ergodic system. In: Classical quantum models and arithmetic problems. Lecture Notes in Physics and Mathematics, Vol. 92. New York: Dekker 1984

16. Klingenberg, W.: Eine Vorlesung über Differentialgeometrie. Berlin, Heidelberg, New York: Springer 1973

17. Klingenberg, W.: Lectures on closed geodesics. Grundlehren der mathematischen Wissenschaften, Vol. 230. Berlin, Heidelberg, New York: Springer 1978

18. Markus, L., Meyer, K.R.: Generic Hamiltonian dynamical systems are neither integrable nor ergodic. Mem. Am. Math. Soc. 144 (1974)

19. Massey, W.S.: Algebraic topology. Graduate Texts in Mathematics, Vol. 56. Berlin, Heidelberg, New York: Springer 1977

20. Milnor, J.: Morse theory. Annals of Mathematics Studies. Princeton: Princeton University Press 1973 
21. Ornstein, D.S.: What does it mean for a mechanical system to be isomorphic to the Bernoulli flow? In: Dynamical systems, theory and applications. Moser, J. (ed.). Lecture Notes in Physics, Vol. 38. Berlin, Heidelberg, New York: Springer 1974

22. Ornstein, D., Weiss, B.: Geodesic flows are Bernoullian. Isr. J. Math. 14, 184-198 (1973)

23. Ratner, M.: Markov partitions for Anosov flows on $n$-dimensional manifolds. Isr. J. Math. 15, 92-114 (1973)

24. Ratner, M.: The central limit theorem for geodesic flows on $n$-dimensional manifolds of negative curvature. Isr. J. Math. 16, 181-197 (1973)

25. Satake, I.: On a generalization of the notion of a manifold. Proc. Nat. Acad. Sci. 42, 359-363 (1956)

26. Satake, I.: The Gauss-Bonnet theorem for $V$-manifolds. J. Math. Soc. Jpn. 9, 464492 (1957)

27. Schwartz, J.T.: Nonlinear functional analysis. New York: Gordon and Breach 1969

28. Siegel, C.L., Moser, J.K.: Lectures on celestial mechanics. Grundlehren der mathematischen Wissenschaften, Vol. 187. Berlin, Heidelberg, New York: Springer 1971

29. Spivak, M.: Differential geometry, Vol. II. Berkeley: Publish or Perish 1979

30. Stiefel, E.L., Scheifele, G.: Linear and regular celestial mechanics. Grundlehren der mathematischen Wissenschaften, Vol. 174. Berlin, Heidelberg, New York: Springer 1971

31. Walters, P.: An introduction to ergodic theory. Graduate Texts in Mathematics, Vol. 79. Berlin, Heidelberg, New York: Springer 1982

Communicated by J. Mather

Received July 26, 1985; in revised form December 8, 1986 